\title{
Effectiveness of Selected Exercise Programme on Cervical Range of Motion in Patients with Thoracic Kyphosis \& Forward Head Posture
}

\section{Monika Dhiman ${ }^{1}$, Maman Paul ${ }^{* 2}$.}

${ }^{1}$ MPT-Orthopedics, Department of Physiotherapy, Guru Nanak Dev University, Amritsar, Punjab, India.

${ }^{* 2}$ Assistant Professor, Department of Physiotherapy, Guru Nanak Dev University, Amritsar, Punjab, India.

\section{ABSTRACT}

Background: Altered postural behaviours result in Forward head posture and thoracic kyphosis making it amenable to correction. The biomechanical strain, in presence of reduced strength of the core stabilizing musculature, in particular, if it is repeated or prolonged, is the predominant explanation for symptoms associated with forward head posture and thoracic kyphosis i.e., neck pain and reduced cervical range of motion.

Objective: The aim of the present study was to investigate and compare the effect of postural awareness and conventional exercises on the cervical range of motion in patients with thoracic kyphosis and forward head posture.

Methodology: This experimental study was conducted on 60 subjects both male and female of age group 20-35yrs. Subjects were randomly divided into two groups consisting of 30 subjects each. Group A received hot pack and postural advice and Group $B$ received hot pack and stretching and strengthening exercises. All the subjects received a total intervention of 4 days (alternate days) per week for 4 weeks.

Results: Intra-group significant differences were obtained between pre- and post-treatment for all evaluated variables ( $\mathrm{pA} 0.01$ ) in both groups. The inter-group comparison showed significant differences (pÂ0.01) between post-treatment variables of Group A and Group B where, Group B showed greater improvement than Group A.

Conclusion: The treatment given to both the groups together can be used to improve cervical range of motion, thoracic kyphosis, and forward head posture. This study may serve as a guideline for physiotherapists when making decisions regarding possible interventions.

KEY WORDS: Cervical range of motion, Craniovertebral angle, Forward head posture, Kyphosis index, Neck disability index, Thoracic kyphosis.

Address for correspondence: Dr. Maman Paul PhD. (Assistant Professor), Department of Physiotherapy, Guru Nanak Dev University, Amritsar, Punjab- 143005, India.

E-Mail: mamanpaul02@gmail.com

Access this Article online

Quick Response code

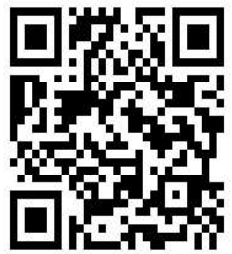

DOI: $10.16965 /$ ijpr.2021.125

\section{Journal Information}

International Journal of Physiotherapy and Research

ISSN (E) 2321-1822 | ISSN (P) 2321-8975

https://www.ijmhr.org/ijpr.html

DOI-Prefix: https://dx.doi.org/10.16965/ijpr

\section{Article Information}

Received: 19 Mar 2021

Peer Review: 20 Mar 2021

Revised: 02 Jun 2021
Accepted: 11 Jun 2021

Published (O): 11 Jul 2021

Published (P): 11 Aug 2021

\section{INTRODUCTION}

Forward head posture (FHP) is a head-on-trunk misalignment and is described (in sitting or standing) as 'any alignment in which the external auditory meatus is positioned anterior to the plumb line through the shoulder joint [1]. According to the literature, FHP changes the biomechanical stress on the 
cervical spine and leads to musculoskeletal disorders for instance cervical pain, headache, temporomandibular and muscular dysfunctions [2].

This posture encompasses weakness in the mid-thoracic scapular retractors (i.e., rhomboids, serratus anterior, middle and lower fibers of the trapezius) and deep cervical short flexor muscles and shortening of the opposing cervical extensors and pectoralis muscles (known as the upper crossed postural syndrome) [3]. Although there is a unanimity that the prolonged adoption of FHP can result in this muscle results from habitual postures assumed over time (e.g., working postures), thus making it amenable to correction through exercise [4]. In FHP the centre of gravity of the head is anterior to the vertical axis (often measured by a plumb line), thereby increasing the load on posterior neck muscles. This biomechanical strain, in the presence of reduced strength of the core stabilizing neck musculature, in particular if it is repeated or prolonged, is the predominant explanation for symptoms associated with FHP [5]. It has been shown by the previous studies that forward head posture results in shortening of the posterior neck extensors, tightening of the anterior neck and shoulder muscles, and affects scapular position and kinematics. In addition to muscle imbalance, FHP has been linked to pain, fatigue and restricted movement of the neck along with symptoms attributed to excessive joint and muscle loading [6].

Excessive thoracic curvature of the spinal column (Hyper kyphosis deformity) is one of the most common postural abnormalities and is one of the most important causes of the upper quarter pathology [7]. Spinal abnormalities in individuals with completed skeletal growth have been reported at an estimated $32 \%$ prevalence in adults and a prevalence of $60 \%$ in the elderly [8]. Early detection, correct assessment, sufficient cure and rehabilitation, prevention and suitable active exercises may impede effects of postural abnormalities. Although improvement in postural alignment secondary to exercise would be expected due to improvement in muscle length and strength, the influence of self-awareness of posture must also be taken into consideration. There is widespread inclusion of postural correction in therapeutic interventions but experimental data to support its effectiveness is limited [9].

Early detection, correct assessment, sufficient cure and rehabilitation, prevention, and suitable active exercise may impede different effect of postural and spinal deformities.

To some extent, postural correction is under our conscious control, so a program that has postural assessment and exercises specially designed to improve posture could increase the postural awareness of participants and potentially change their habitual postures. Despite of the fact that widespread inclusion of postural correction in therapeutic interventions is there, experimental data to support its effectiveness is limited [9].

\section{METHODS}

The present study was experimental in nature. The study was done in the Department of Physiotherapy, Guru Nanak Dev University, Amritsar.

Ethical Approval and Consent: Before the study began, ethical approval was obtained from the institutional ethical committee (vide letter No. (225/HG) dated 27/09/2018). Prior to the commencement of the study, each participant was explained the purpose, aims, objectives and risks associated with the study and thereafter their written consent was obtained.

Participants: Sixty adults (mean [SD] age $=27.95 \pm 4.24$ years), both male and female were included and divided into 2 groups using random sampling technique. Subjects having forward head posture and thoracic kyphosis, cervical pain- with/without referred pain, numbness or paraesthesia, pain and functional difficulty of some extent for at least 1 week participated. Subjects were excluded if they had a history of spinal or lower limb fractures, neuromuscular disorder, moderate and severe scoliosis, visual impairment not corrected by prescriptive lenses, whiplash injury, dizziness, foot deformities, history of falls over the past one year, or significant impaired function due to their lower back or lower limbs that would overshadow their neck pain and 
affect their standing posture.

Anthropometric Variables: Three anthropometric variables namely height, weight and BMI were measured from all the subjects using the standard techniques [1] and were measured in triplicate with the median value used as the criterion. Stadiometer (Holtain Ltd. Crymych, Dyfed, UK) was used for measuring standing height. Subjects were weighed in minimal light-weight clothing, bare foot, using standard weighing machine (Model DS-410, Seiko, Tokyo, Japan) to the nearest $0.1 \mathrm{~kg}$. Body mass index (BMI) was calculated from height and weight as follows: BMl=weight $(\mathrm{kg}) /$ height2 (m2).

\section{Treatment Protocol}

Group A: Group received hot pack for $15 \mathrm{~min}$ and Postural advice. Patients had to follow the postural advice throughout the four week session and had to perform the advised changes during their usual work routine. It included: 1. To Decrease a Forward Head Posture: [10] (a) Axial Extension (Cervical Retraction) and (b) Scapular Retraction and 2. Stress Management/Relaxation: (a) Cervical and Upper Thoracic Region and (b) Lower Thoracic Region

Table A: Treatment protocol for Forward Head Posture [5]:

\begin{tabular}{|l|c|c|c|c|}
\hline \multicolumn{1}{|c|}{ Exercises } & Progression 1 & Progression 2 & Progression 3 & Progression 4 \\
\hline Strengthen Deep Cervical Flexors & Lying chin tuck & $\begin{array}{c}\text { Lying chin tuck with } \\
\text { head lift }\end{array}$ & Lying chin tuck with head lift* & Lying chin tuck with head lift* \\
\hline Stretch Cervical Extensors & Chin drop & $\begin{array}{c}\text { Chin drop with hand } \\
\text { assistance }\end{array}$ & Chin drop with hand assistance \\
\hline Strengthen Shoulder Retractors & $\begin{array}{c}\text { Standing shoulder pull } \\
\text { back with elastic } \\
\text { resistance }\end{array}$ & $\begin{array}{c}\text { Shoulder pull back with } \\
\text { weight Left and Right } \\
\text { side }\end{array}$ & $\begin{array}{c}\text { Shoulder pull back with elastic } \\
\text { resistance and weight Left } \\
\text { and Right side }\end{array}$ & $\begin{array}{c}\text { Shoulder pull back with elastic } \\
\text { resistance and weight Left and } \\
\text { Right side }\end{array}$ \\
\hline Stretch Pectrolis Muscle & $\begin{array}{c}\text { Pectoral stretch Left } \\
\text { and Right side }\end{array}$ & Bilateral Pectoral stretch & $\begin{array}{c}\text { Pectoral stretch Left and Right } \\
\text { side }\end{array}$ & Bilateral Pectoral stretch \\
\hline
\end{tabular}

*Progressions were done with 2-second hold starting at 2 seconds i.e., 2, 4, and 6 seconds. (In some cases, if patients were unable to progress by 2 seconds, but rather by 1 second)

Group B: Group received hot pack for $15 \mathrm{~min}$ and exercises for forward head posture and thoracic kyphosis. The treatment program was performed four days per week (Alternate days) for four weeks. Each treatment session lasted for about an hour. (Table A and Table B)

Table B: Treatment protocol of Thoracic Kyphosis [10].

\begin{tabular}{|l|c|c|c|c|}
\hline \multicolumn{1}{|c|}{ Exercises } & Progression 1 & Progression 2 & Progression 3 & Progression 4 \\
\hline Stretch upper thoracic region* & Pectoralis major stretch & $\begin{array}{c}\text { Increase flexibility of } \\
\text { anterior thorax }\end{array}$ & $\begin{array}{c}\text { Pectoralis major } \\
\text { stretch }\end{array}$ & $\begin{array}{c}\text { Increase flexibility of } \\
\text { anterior thorax }\end{array}$ \\
\hline $\begin{array}{l}\text { Extension exercises for lower } \\
\text { thoracic spine** }\end{array}$ & Prone press-up & Prone Press-up & Prone Press-up & Prone Press-up \\
\hline
\end{tabular}

*Stretch held for 30 seconds and 3 sets of 12 repetitions.

**3 sets of 12 repetitions starting at 5 seconds and progressed by 5 -second holds i.e., 10, 15, and 20 seconds. The initial hold time of the stretch was dependent on the patient's ability.

\section{Outcome measures:}

Neck disability: The Neck disability index (NDI) is a condition-specific disability measure. The NDI contains 10 items ( 7 related to activities of daily living, 2 related to pain, and 1 item related to concentration). Score of each item is ranges 0 to 5 and the total score is expressed as a percentage, with higher scores represents the greater disability [11].

Range of motion: Neck Range of motion was measured in a sitting position to remove errors and movement compensation. A large
Universal goniometer was used for measuring the range of motion. The range of motion in all six directions was measured [12].

Thoracic kyphosis- Kyphosis index: Thoracic kyphosis was measured using the flexicurve method. Subjects were instructed to stand in their usual posture whilst we place the flexicurve over the spinous processes of the thoracic and lumbar spine. The ends of the flexicurve aligning according to $\mathrm{C} 7$ and S2 spinous processes and the shape of the flexicurve conform the curvature of the spine. 
Next, the flexicurve was carefully placed on paper and its outline traced. A straight line drawn from the ruler position of C7 to S2 that corresponded to the length of thoracic kyphosis calculated as described below. The kyphosis index is calculated from thoracic width (B) divided by the horizontal length (L) multiplied by 100 (B/L x 100) (Fig.1). In our study, three measurements were obtained and the average was used in the data analysis [13].

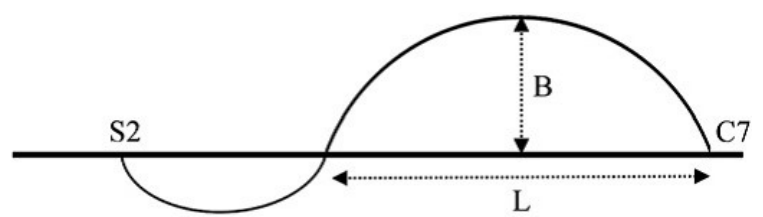

Fig. 1: Representation of measurement of kyphosis Index.

Forward head posture: A digital imaging technique was used to evaluate FHP posture in the standing position. A digital camera (Samsung SM-A307FN) was used. The height of the camera was adjusted to the level of the subject's shoulder and a self- balanced position was chosen to standardize the head and neck posture of subjects. The necessity of remaining in natural posture during taking photographs was explained by the assessor. Lateral-view photographs of the subject in his/her usual standing posture were used for

\begin{tabular}{lcccccc}
\hline \multirow{2}{*}{ Variables } & \multicolumn{2}{c}{ Group A } & \multicolumn{2}{c}{ Group B } & \multirow{2}{*}{ t-value } & \multirow{2}{*}{$\boldsymbol{p}$-value } \\
\cline { 2 - 5 } & Mean & SD & Mean & SD & & \\
\hline Age (years) & 26.93 & 4.44 & 28.96 & 3.82 & 1.898 & $0.63^{*}$ \\
Height (cm) & 164.5 & 8.32 & 164.76 & 6.75 & 0.136 & $0.892^{*}$ \\
Weight (Kg) & 59.1 & 9.68 & 58.83 & 8.87 & 0.111 & $0.912^{*}$ \\
BMl (Kg/m2) & 21.7 & 2.29 & 21.8 & 2.29 & 0.112 & $0.911^{*}$ \\
*Non-significant $(P>0.05)$ & & & & & \\
\hline
\end{tabular}

SD: standard deviation; $p$-value: probability value; Significant at level $(p<0.05)$

Rt. = Right; Lt. = Left; BMI= Body mass index

Table 2: Descriptive statistics of different pre-treatment variables (Inter-group) of Group A and Group B.

\begin{tabular}{lcccccc}
\hline \multirow{2}{*}{ Variables } & \multicolumn{2}{c}{ Group A } & \multicolumn{2}{c}{ Group B } & \multirow{2}{*}{ t-value } & \multirow{2}{*}{$\boldsymbol{p}$-value } \\
\cline { 2 - 5 } & Mean & SD & Mean & SD & & \\
\hline AFROM (Degree) & 36.03 & 3.45 & 35.3 & 3.15 & 0.858 & $0.394^{*}$ \\
\hline AEROM (Degree) & 45 & 3.57 & 45.3 & 4.09 & 0.302 & $0.763^{*}$ \\
\hline ALF(Rt) (Degree) & 35.33 & 3.05 & 35.36 & 2.85 & 2.575 & $0.364^{*}$ \\
\hline ALF(Lt) (Degree) & 36 & 2.8 & 35.16 & 3.41 & 1.033 & $0.306^{*}$ \\
\hline ARROM(Rt) (Degree) & 63.7 & 4.52 & 63.6 & 3.04 & 0.095 & $0.636^{*}$ \\
\hline ARROM(Lt) (Degree) & 61.4 & 4.78 & 61.56 & 4.66 & 4.232 & $0.712^{*}$ \\
\hline KI & 21.42 & 2.4 & 21.19 & 2.04 & 0.4 & $0.691^{*}$ \\
\hline CVA (Degree) & 37.63 & 2.8 & 37.7 & 2.91 & 0.09 & $0.928^{*}$ \\
\hline NDI & 36.23 & 2.62 & 36.2 & 2.51 & 0.05 & $0.960^{*}$ \\
\hline
\end{tabular}

*Non-significant $(p>0.05)$

Rt. = Right; Lt. = Left; NDI= Neck Disability Index; AFROM= Active flexion range of motion; AEROM= Active extension range of motion; $A L F=$ Active lateral flexion; $A R R O M=$ Active rotation range of motion; $\mathrm{KI}=\mathrm{Kyphosis} \mathrm{Index;}$ $\mathrm{CVA}=$ Craniovertebral angle; NDI= Neck disability index 
Table 3: Descriptive statistics of different variables between pre-treatment and post-treatment of Group A:

\begin{tabular}{|c|c|c|c|c|c|c|c|}
\hline \multirow{2}{*}{ Variables } & \multicolumn{2}{|c|}{ Pre-treatment } & \multicolumn{2}{|c|}{ Post-treatment } & \multirow{2}{*}{$\mathrm{t}$-value } & \multirow{2}{*}{$p$-value } & \multirow{2}{*}{$\begin{array}{l}\text { Percentage } \\
\text { increment }\end{array}$} \\
\hline & Mean & SD & Mean & SD & & & \\
\hline AFROM (Degree) & 36.03 & 3.45 & 40.9 & 3.11 & 28.445 & $0.001 * * *$ & $13.51 \%$ \\
\hline AEROM (Degree) & 45 & 3.57 & 49.4 & 2.59 & 12.775 & $0.001^{* * *}$ & $9.77 \%$ \\
\hline ALF(Rt) (Degree) & 35.33 & 3.05 & 38.7 & 2.36 & 7.089 & $0.001 * * *$ & $9.53 \%$ \\
\hline ALF(Lt) (Degree) & 36 & 2.8 & 38.7 & 2.32 & 11.012 & $0.001 * * *$ & $7.50 \%$ \\
\hline ARROM(Rt) (Degree) & 63.7 & 4.52 & 70.8 & 3.06 & 15.327 & $0.001 * * *$ & $11.10 \%$ \\
\hline ARROM(Lt) (Degree) & 61.4 & 4.78 & 67.66 & 3.85 & 18.137 & $0.001 * * *$ & $10.19 \%$ \\
\hline KI & 21.42 & 2.4 & 18.19 & 2.07 & 20.818 & $0.001^{* * *}$ & $17.75 \% *$ \\
\hline CVA (Degree) & 37.63 & 2.8 & 42.16 & 2.5 & 21.845 & $0.001 * * *$ & $12.03 \%$ \\
\hline NDI & 36.23 & 2.62 & 27.86 & 4.63 & 17.016 & $0.001 * * *$ & $30.04 \% *$ \\
\hline
\end{tabular}

SD: standard deviation; $p$-value: probability value; Significant at level $(p<0.05)$

Rt. = Right; Lt. = Left; NDI= Neck Disability Index; AFROM= Active flexion range of motion; AEROM= Active extension

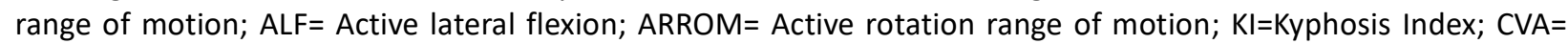
Craniovertebral angle; NDI= Neck disability index

Table 4: Descriptive statistics of different variables between pre-treatment and post-treatment of Group B:

\begin{tabular}{lccccccc}
\hline \multirow{2}{*}{ Variables } & \multicolumn{2}{c}{ Pre-treatment } & \multicolumn{2}{c}{ Post-treatment } & \multirow{2}{*}{ t-value } & $\boldsymbol{p}$-value & $\begin{array}{c}\text { Percentage } \\
\text { increment }\end{array}$ \\
\cline { 2 - 5 } & Mean & SD & Mean & SD & & & \\
\hline AFROM (Degree) & 35.3 & 3.15 & 44.46 & 2.94 & 23.171 & $0.001^{* * *}$ & $25.94 \%$ \\
AEROM (Degree) & 45.3 & 4.09 & 54.9 & 3.5 & 20.267 & $0.001^{* * *}$ & $21.19 \%$ \\
ALF(Rt) (Degree) & 35.36 & 2.85 & 42.06 & 1.77 & 16.267 & $0.001^{* * *}$ & $24.60 \%$ \\
ALF(Lt) (Degree) & 35.16 & 3.41 & 42.46 & 1.67 & 9.744 & $0.001^{* * *}$ & $20.76 \%$ \\
ARROM(Rt) (Degree) & 63.6 & 3.04 & 77.16 & 2.45 & 19.156 & $0.001^{* * *}$ & $21.32 \%$ \\
ARROM(Lt) (Degree) & 61.56 & 4.66 & 76.23 & 2.81 & 21.213 & $0.001^{* * *}$ & $14.52 \%$ \\
KI & 21.19 & 2.04 & 13.64 & 1.57 & 23.248 & $0.001^{* * *}$ & $55.35 \% *$ \\
CVA (Degree) & 37.7 & 2.91 & 46.96 & 2.96 & 17.223 & $0.001^{* * *}$ & $24.56 \%$ \\
NDI & 36.2 & 2.51 & 23.8 & 2 & 56.407 & $0.001^{* * *}$ & $52.1 \% *$ \\
\hline
\end{tabular}

***Highly significant $(p \leq 0.001) *$ Percentage Decrement

SD: standard deviation; $p$-value: probability value; Significant at level $(p<0.05)$

Rt. = Right; Lt. = Left; NDI= Neck Disability Index; AFROM= Active flexion range of motion; AEROM= Active extension range of motion; $A L F=$ Active lateral flexion; $A R R O M=$ Active otation range of motion; KI=Kyphosis Index; $\mathrm{CVA}=$ Craniovertebral angle; $\mathrm{NDI}=$ Neck disability index

\begin{tabular}{lcccccc}
\hline \multirow{2}{*}{ Variables } & \multicolumn{2}{c}{ Group A } & \multicolumn{2}{c}{ Group B } & \multirow{2}{*}{ t-value } & p-value \\
\cline { 2 - 5 } & Mean & SD & Mean & SD & & \\
\hline AFROM (Degree) & 40.9 & 3.11 & 44.46 & 2.94 & 4.56 & $0.001^{* * *}$ \\
AEROM (Degree) & 49.4 & 2.59 & 54.9 & 3.5 & 6.905 & $0.002^{* *}$ \\
\hline ALF(Rt) (Degree) & 38.7 & 2.36 & 42.06 & 1.77 & 6.229 & $0.001^{* * *}$ \\
ALF(Lt) (Degree) & 38.7 & 2.32 & 42.46 & 1.67 & 7.205 & $0.001^{* * *}$ \\
ARROM(Rt) (Degree) & 70.8 & 3.06 & 77.16 & 2.45 & 8.882 & $0.001^{* * *}$ \\
ARROM(Lt) (Degree) & 67.66 & 3.85 & 76.23 & 2.81 & 9.835 & $0.002^{* *}$ \\
KI & 18.19 & 2.07 & 13.64 & 1.57 & 9.542 & $0.001^{* * *}$ \\
CVA (Degree) & 42.16 & 2.5 & 46.96 & 2.96 & 6.772 & $0.001^{* * *}$ \\
\hline NDI & 27.86 & 2.94 & 23.8 & 3.08 & 5.219 & $0.001^{* * *}$ \\
\hline ***Highly significant $(p \leq 0.001)$ & $* *$ Significant $(p \leq 0.01)$ & & & & \\
\hline
\end{tabular}

SD: standard deviation; $p$-value: probability value; Significant at level $(p<0.05)$

Rt. = Right; Lt. = Left; NDI= Neck Disability Index; AFROM= Active flexion range of motion; AEROM= Active extension range of motion; $A L F=$ Active lateral flexion; $A R R O M=$ Active rotation range of motion; KI=Kyphosis Index; $\mathrm{CVA}=$ Craniovertebral angle; $\mathrm{NDI}=$ Neck disability index

Table 1 shows the Descriptive statistics of anthropometric variables (age, height, weight and BMI) in Group A and Group B. No significant difference was noted in any case.

Table 2 shows the descriptive statistics of different (Inter-group) pre-treatment variables of Group A and Group B. No statistically significant differences were noted.

Table 3 and Table 4 shows within-group (intra-group) analysis of Group A and Group B respectively, in which both interventions showed significant differences at post-treatment $(p<0.05)$.

Table 5 shows inter-group analysis (Group A 
vs. Group B) with respect to variables at post-treatment measurement. Statistically significant differences $(p<0.05)$ were noted.

\section{DISCUSSION}

This study compared the effects of Postural awareness and stretching and strengthening exercises in increasing cervical ROM in subjects with thoracic kyphosis and forward head posture and the results showed that there was significant $(p<0.05)$ improvement in the same followed by both the procedures as well as significant $(p<0.05)$ difference between the two procedures. The results of this study are in line with some previous studies. Quintero $Y$ et al., 2009 stated in his study that 'Awareness through Movement' (ATM) is a process, which facilitates the learning of strategies for improving organization and coordination of body movement by developing spatial and kinaesthetic awareness of body-segment relationships at rest and during motion. Through the specific use of sensorimotor experiences, the Awareness through Movement (ATM) purports to enhance people's awareness of their habitual solutions to motor problems and the sensations accompanying those habits. Anticipatory postural adaptations enhance postural control and can be used in postural correction [14]. The strengthening of the back extensor muscles and the muscles around the spine has a significant effect on the reduction of kyphosis angle [15].

From the study we inferred that a treatment program involving both aspects that is, postural awareness and stretching and strengthening exercises would improve the forward head posture and thoracic kyphosis along-with improvement in the cervical range of motion. Exercise programs with elastic bands, which are easily accessible, can be used effectively in the correction of posture, without temporal and spatial limitations [16]. In our study, we used theraband for strengthening of the shoulder retractors that helped in improvement of forward head posture and thoracic kyphosis that further improved cervical range of motion. Liu S et al., 2016 stated in his study that for cervical ROM, the group had a significant increase $\left(3.7^{\circ}\right)$ in flexion, as compared to the $C$ group at the end of the study. Two exercises targeted this movement, and our findings suggest that the combination of stretching (chin drop) and strengthening (chin tuck) effected this change.

Forward head posture is diagnosed when the Craniovertebral angle is less than $50^{\circ}$ [16]. When comparing the craniovertebral angle measured before and after the treatment program, we found that it was increased by $12.03 \%$ in group $A$ and $24.56 \%$ in group $B$. Kyphosis index in normal adults ranges from 3-13 [17]. In our study when we compared the kyphosis index measured before and after the treatment program, we found that the kyphosis index decreased by $17.75 \%$ in group $A$ and $55.35 \%$ in group $B$.

Limitations of this study: A limited number of subjects in the study. The duration of intervention was four weeks only. There was no control group in the study.

\section{CONCLUSION}

The results of this study supported the previous studies which studied the effect of postural awareness and stretching and strengthening exercises in forward head posture and thoracic kyphosis and concluded that these exercises had great effects on cervical range of motion and neck disability. Ours is a positive finding with respect to the education and awareness component of postural programs and should inform all exercise programs that the postural awareness along with stretching and strengthening program should be engaged in any postural re-education program.

\section{ABBREVIATIONS}

FHP - Forward Head Posture

NDI - Neck Disability Index

BMI - Body Mass Index

CVA - Craniovertebral Angle

ATM - Awareness Through Movement

\section{ACKNOWLEDGEMENT}

The authors would like to thanks the subjects who voluntarily participated in the study; none of this would have been possible without them. 


\section{Conflicts of interest: None}

\section{REFERENCES}

[1]. Kendall F and McCreary E. Muscles: Testing and Function with Posture and Pain. $5^{\text {th }}$ ed. PA: Lippincott Williams and Wilkins; 2005.

[2]. Lau M, Wing T \& Lam $H$. The effectiveness of thoracic manipulation on patients with chronic mechanical neck pain-a randomized controlled trial. Man Ther 2011; 16(2): 141-7.

[3]. Janda V. Muscles and motor control in cervicogenic disorders: Assessment and management. Physical therapy of the cervical and thoracic spine. $2^{\text {nd }}$ ed. New York: Churchill Livingstone; 1994. p. 195-216.

[4]. Mense S, Simons DG. Reflexly mediated and postural muscle pain. eds. Muscle Pain: Understanding its Nature, Diagnosis and Treatment. Philadelphia, PA: Lippincott Williams \& Wilkins 2001: 131157.

[5]. Harman K, Hubley L \& Butler H. Effectiveness of an exercise program to improve forward head posture in normal adults: a randomized, controlled 10week trial. J Manual Manip Ther 2005; 13(3): 163176.

[6]. Griegel-Morris P, Larson K, Mueller-Klaus K, Oatis C. Incidence of common postural abnormalities in the cervical, shoulder and thoracic regions and their association with pain in two age groups of health subjects. Phys Ther 1992; 72: 425-431.

[7]. Hertling D, Kessler RM. Management of common musculoskeletal disorders: physical therapy principles and methods. $4^{\text {th }}$ ed. Philadelphia: Lippincott Williams \& Wilkins; 2006.

[8]. Liu S, Diebo BG, Henry JK, Smith JS, Hostin R, Cunningham ME. et al. The benefit of non-operative treatment for adult spinal deformity: identifying predictors for reaching a minimal clinically important difference. Spine J 2016; 16: 210-18.

[9]. Katzman WB, Vittinghoff E, Lin F, Schafer A, Long RK, Wong S, Gladin A, Fan B, Allaire B, Kado DM, Lane NE. Targeted spine strengthening exercise and posture training program to reduce hyperkyphosis in older adults: results from the study of hyperkyphosis, exercise, and function (SHEAF) randomized controlled trial. Osteoporos Int 2017; 28 : 2831-2841.
[10]. Kisner C. Therapeutic Exercise: Foundations and techniques $7^{\text {th }}$ Edition; 2018.

[11]. Cleland, J. A., Fritz, J. M., Whitman, J. M., \& Palmer, J. A. The Reliability and Construct Validity of the Neck Disability Index and Patient Specific Functional Scale in Patients with Cervical Radiculopathy. Spine 2006; 31(5): 59-602.

[12]. Farooq M, Khan G. Reliability of the universal goniometer for assessing active cervical range of motion in asymptomatic healthy persons. Pak J Med Sci 2016; 32(2): 457-461.

[13]. June Q, Yong-Hao P, Ross A.C, Adam L.B. Effects of thoracic kyphosis and forward head posture on cervical range of motion in older adults. Man Ther 2012; 18(1): 65-71.

[14]. Marcio J.S, Neeta K, Alenxander S. A. The role of anticipatory postural adjustments in compensatory control of posture. J Electromyogr Kinesiol 2010; 20(3): 388-397.

[15]. Zabiholah T, Reza R, Hooman M, Shahnaz S. The effect of spine strengthening exercises and posture training on Functional Thoracic Hyper Kyphosis in young indiviuals. J Adv Med Biomed Res 2019; 27(121): 23-31.

[16]. Tae-Woon K, Da-In A, Hye-Yun L, Ho-Young J, DongHyun K. Effects of elastics band exercise on subjects with rounded shoulder posture and forward head posture. The J Physi Ther Sci 2016; 28: 17331737.

[17].Barrett E, McCreesh K, Lewis J. Intrarater and Interrater Reliability of the Flexicurve Index, Flexicurve Angle, and Manual Inclinometer for the Measurement of Thoracic Kyphosis. Rehabil Res Pract Volume 2013; Article ID 475870.
How to cite this article:
Monika Dhiman, Maman Paul. Effectiveness of Selected Exercise
Programme on Cervical Range of Motion in Patients with Thoracic
Kyphosis \& Forward Head Posture. Int J Physiother Res 2021;9(4):3863-
3869. DOI: 10.16965/ijpr.2021.125 